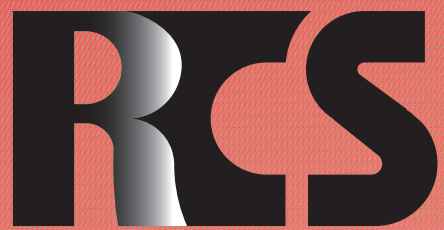

Depósito legal ppi $201502 Z U 4662$

Esta publicación científica en formato digital es continuidad de la revista impresa Depósito Legal: pp $197402 Z U 789$

- ISSN: 1315-9518 • ISSN-E: 2477-9431

Revista de Ciencias Sociales

Universidad del Zulia. Revista de la Facultad de Ciencias Económicas y Sociales Vol. XXVII. No. 2

Abril-Junio 2021

Esta publicación científica en formato digital es continuidad de la revista impresa Depósito Legal: pp $197402 Z$ Z789 ISSN: 1315-9518 


\title{
COVID-19, educación en emprendimiento e intenciones de emprender: Factores decisorios en estudiantes universitarios
}

\author{
Martínez Garcés, Josnel* \\ Durán Omaña, Sandra** \\ Serna Borja, Walter ${ }^{* * *}$
}

\section{Resumen}

El objetivo de esta investigación fue analizar las intenciones emprendedoras de estudiantes universitarios en un entorno vulnerado por las consecuencias derivadas de la COVID-19. Bajo un método positivista, deductivo-cuantitativo, analítico, co-relacional, no experimental y de campo se abordaron 152 estudiantes de una institución de educación superior ubicada en el departamento Valle del Cauca Colombia que estuvieron cursando asignaturas de emprendimiento durante el período académico 2020-02. Los resultados evidencian que $31 \%$ de ellos tiene un emprendimiento activo y $38 \%$ tiene la intención de iniciar uno. 13,33\% consideran que la educación recibida en emprendimiento dentro de la institución fue su inspiración para materializar esta iniciativa y $54,29 \%$ reconoce que fue de gran ayuda. Entre las principales intenciones emprendedoras destacan autoconfianza $(\mu: 3,16)$, creatividad $(\mu: 3,12)$, capacidades y habilidades $(\mu: 3,10)$ y necesidades económicas $(\mu: 3,00)$. Se concluye, que la educación en emprendimiento sí resulta efectiva en la consolidación de las ideas de negocios de los jóvenes universitarios y que las intenciones determinantes van enfocadas a aspectos de índole personal, de formación y del entorno; se destaca el efecto positivo que esto ha tenido entre los jóvenes encuestados quienes, a pesar del contexto sanitario mundial, encuentran en el emprendimiento una alternativa sostenible de empleabilidad, ingreso y bienestar.

Palabras clave: COVID-19; educación; emprendimiento; estudiantes universitarios; intenciones emprendedoras.

\footnotetext{
Magister Scientiarum en Planificación y Gerencia de Ciencia y Tecnología. Magister Scientiarum en Gerencia de Empresas, mención Gerencia Financiera. Coordinador de Investigación en la Corporación Universitaria Autónoma de Nariño, Colombia. E-mail: martinezjosnel@gmail.com iD ORCID: https://orcid.org/0000-0002-8120-3285

** MBA en Administración y Dirección de Empresas. Coordinadora del programa Ciencias Económicas, Administrativas y Contables en la Corporación Universitaria Autónoma de Nariño, Colombia. E-mail: smile.leidenz@,gmail.com iD ORCID: https://orcid.org/0000-0002-2447-7677

*** Magister en Economía. Docente-Investigador en la Corporación Universitaria Autónoma de Nariño, Colombia. E-mail: wasebo76@hotmail.com iD ORCID: https://orcid.org/0000-0003-2083-4806
} 


\title{
COVID-19, entrepreneurship education and intentions to undertake: Decision factors in university students
}

\begin{abstract}
The objective of this research was to analyze the entrepreneurial intentions of university students in an environment affected by the consequences derived from COVID-19. Under a positivist, deductivequantitative, analytical, co-relational, non-experimental and field method, 152 students from a higher education institution located in the Valle del Cauca department - Colombia who were taking entrepreneurship courses during the academic period 2020 were approached. -02 . The results show that $31 \%$ of them have an active entrepreneurship and $38 \%$ intend to start one. $13.33 \%$ consider that the education received in entrepreneurship within the institution was their inspiration to materialize this initiative and $54.29 \%$ recognize that it was of great help. Among the main entrepreneurial intentions, self-confidence $(\mu: 3.16)$, creativity $(\mu: 3.12)$, abilities and skills $(\mu: 3.10)$ and economic needs ( $\mu: 3.00)$ stand out. It is concluded that entrepreneurship education is effective in consolidating the business ideas of young university students and that the determining intentions are focused on aspects of a personal nature, training and the environment; The positive effect that this has had among the young people surveyed is highlighted who, despite the global health context, find entrepreneurship a sustainable alternative for employability, income and well-being.
\end{abstract}

Keywords: COVID-19; education; entrepreneurship; college students; entrepreneurial intentions.

\section{Introducción}

El emprendimiento en Colombia es una práctica ampliamente estudiada a lo largo de las últimas décadas; y no es para menos, puesto que ha experimentado un sobresaliente auge que resulta digno de análisis. Según Tarapuez, Osorio y Botero (2013), la acción pública del Estado sobre este asunto data desde los inicios de los años 2000, momento en el cual algunos funcionarios del gobierno nacional con fuertes vinculaciones al sector empresarial, proponen elevarlo a condición de problema público.

De acuerdo con Pérez y Álvarez (2017), los resultados del Global Entrepreneurship Monitor (GEM) indican que en el país un 70 por ciento de la población adulta (entre 18 y 64 años) considera viable la opción de ser empresario como un estilo de vida profesional, además de aportarles reconocimiento social. A razón de esto, la adecuada orientación de su dinámica productiva, es un impulso al desarrollo global en un entorno cada vez más competitivo (Patiño, Ruiz y Pitre-Redondo,
2018).

Sin embargo, como todo proceso humano y social, la práctica de emprender se encuentra rodeada de algunas dificultades que, como sostienen Díaz y Silva (2017), al ser identificadas oportunamente pueden convertirse en retos susceptibles de propuestas transformadoras, tanto desde el imaginario individual y colectivo así como a la luz de políticas estatales e institucionales.

Uno de los principales brotes en los últimos años (por no decir que el más grande y complejo de todos), ha sido la pandemia mundial a razón de la COVID-19. Este nuevo escenario que trastocó todo el sistema político, económico y social de los países, tomó especial interés nacional cuando el Gobierno a través del Ministerio de Salud y Protección Social de la República de Colombia (2020), anuncia oficialmente la detección del primer contagiado en el país el día 06 de marzo de 2020.

Desde entonces, y como medidas de contención sanitaria y preservación de la vida, 
se han implementado distintas estrategias como cuarentenas, toques de queda, restricciones de movilidad, que han ido variando en función del comportamiento de la cadena de contagios en cada localidad. Estas acciones, han traído consigo efectos negativos al sector empresarial y productivo. Se ha podido conocer que tan sólo en Bogotá, la capital del país, durante los primeros seis meses de pandemia un total de 37.000 empresas fueron liquidadas, es decir, $63 \%$ más que el mismo período durante el año anterior. De estas, 99\% eran mipymes: $95 \%$ bajo la figura de personas naturales y $5 \%$ en forma de personería jurídica (Semana, 2020). La misma fuente señala, que los principales sectores afectados han sido los restaurantes, tiendas, bares y peluquerías, los cuáles, por regla general, son producto de emprendimientos.

No obstante, a pesar de lo difícil de esta situación muchos expertos consideran que el país ha entrado en "la nueva era del emprendimiento". Así, para Hinojosa (2021), la pandemia, la informalidad, el desempleo y las limitaciones de movilidad, han incentivado un aumento de ellos. En este orden de ideas, se hizo propicia la aprobación de la Ley 2069, o Ley de emprendimiento, que nace en el seno del Plan Nacional de Desarrollo 2018-2022 y que, a juicio de BC Noticias (2021), se destaca por:

a. Establecer tarifas diferenciadas con simplificación para la creación de emprendimientos.

b. Facilitar el acceso al mercado de compras públicas para mipymes.

c. Orientar la consecución de recursos y acceso a instrumentos financieros.

d. Actualizar el marco normativo alrededor de la institucionalidad para el emprendimiento.

e. Implementar medidas que involucren al sistema educativo con el emprendimiento desde los primeros años de colegio y en la universidad.

Con relación a este último aspecto, la promoción del emprendimiento desde los entornos educativos ya venía dada desde la Ley 1014, publicada en el año 2006, donde se considera que este tipo de formación debe permitir el desarrollo de competencias para hacer frente a las exigencias del sector productivo. Paralelamente, Avendaño, Luna y Quintero (2020), puntualizan que, en el afán de conseguir este propósito, no se debe dar por sentado ni dejar de lado el carácter universal, constructivo, edificante, demócrata y plural, que debe tener la educación en el país.

Así las cosas, este asunto resulta de esencial interés en la presente investigación pues deja entrever cómo, desde una visión normativa a nivel nacional, se resalta la importancia de la formación en emprendimiento. Por ello, este estudio se centra en analizar las intenciones emprendedoras, particularmente de los estudiantes de una institución de educación superior ubicada en el departamento Valle del Cauca - Colombia, en un entorno vulnerado por las consecuencias derivadas de la COVID-19.

\section{Educar para emprender: La asignatura obligatoria}

Según Cardona, Montenegro y Hernández (2017), las universidades están atravesando una transformación de su modelo pedagógico tradicional hacia uno enfocado en el fortalecimiento de competencias y fomento de la actitud emprendedora. Esta nueva propuesta se orienta hacia tres tipos de beneficios: El personal (lucro individual), el social (generación de empleos) y el colectivo (oferta de productos y servicios). Sin embargo, en su adherencia a la figura de libertad en la organización curricular, algunas instituciones aún se han quedado rezagadas en estos esfuerzos (Rico y Santamaría, 2017).

En este contexto, Muñóz y Martínez (2020) junto a Suárez, Suárez y Zambrano (2017), consideran que el emprendimiento promovido entre los jóvenes en formación puede ser un garante de la innovación social expresada en una riqueza que se concreta en la satisfacción del bien común. Cuando desde la formación se tienen sólidas bases en emprendimiento, resulta más fácil identificar 
oportunidades y mantener una actitud favorable frente a las inestabilidades del entorno (Durán y Martínez, 2019; Contreras y Macías, 2021; Solis, et al., 2021).

Para los estudiantes colombianos, la acción de emprender se asocia a calidad de vida (fundamentada en estabilidad y seguridad laboral) y estatus social, puesto que el empresariado goza de un aceptable respeto; de allí, que se estimula a que las universidades promuevan ferias especializadas y capacitaciones específicas para exponer la labor emprendedora como un medio sostenible de ingresos económicos (Rodríguez y Prieto, 2009; Borrayo, Valdez y Delgado, 2019).

Alineado a esto, Arias y Castillo (2011) aseveran que, ante una creciente constante del desempleo y la realidad de que en el país la mayoría de ofertas laborales se generan desde las microempresas y los independientes, ser capaces de emprender es fundamental para el desarrollo profesional y personal. A partir de esta idea, se deriva una contundente afirmación: La educación universitaria tiene una relación directa con el emprendimiento (Chicas, 2020).

De esta manera queda claro que, con las transformaciones globales que el mundo ha experimentado en las últimas décadas y que han incidido en la cosmovisión de los jóvenes contemporáneos, sumándole a ello las afectaciones producto de la COVID-19 desde inicios del año 2020, el fomento del emprendimiento ya no debe ser una opción, sino una necesidad. Los sistemas educativos, deben evolucionar en esa dirección constituyendo esta una apuesta de valor en el intangible de formación que ofrecen a sus estudiantes.

\section{Metodología}

Desde el punto de vista metodológico, la investigación está inmersa en el paradigma positivista con un enfoque deductivocuantitativo. Se tipifica como analítica, corelacional, no experimental y de campo. El universo estuvo conformado por los estudiantes activos de una institución de educación superior, ubicada en el departamento Valle del Cauca - Colombia, que estuvieron cursando asignaturas de emprendimiento durante el período académico 2020-02. El muestreo fue no probabilístico y de participación voluntaria; de esta manera, la muestra final se caracteriza en la Tabla 1.

Tabla 1

Características de la muestra

\begin{tabular}{lcc}
\hline \multicolumn{1}{c}{ Programa } & Cantidad & Representatividad \\
\hline Administración de empresas & 31 & $81 \%$ \\
Contaduría pública & 76 & $84 \%$ \\
Gestión empresarial & 11 & $59 \%$ \\
Ingeniería informática & 3 & $75 \%$ \\
Procesos administrativos & 14 & $100 \%$ \\
Producción gráfica & 17 & $89 \%$ \\
\multicolumn{1}{c}{ Total } & $\mathbf{1 5 2}$ & $\mathbf{8 1 \%}$ \\
\hline
\end{tabular}

Fuente: elaboración propia, 2020.

Para la recolección de los datos, se utilizó la técnica de la encuesta, y como instrumento el cuestionario. Este estuvo integrado por 17 items con opciones de respuestas en escala, de selección simple, y abiertas. Se aplicó durante el último trimestre del año 2020 utilizando la herramienta Google forms. La confiabilidad del mismo se midió a través del cálculo del 
Alfa de Cronbach, el cual arrojó un resultado de 0,912 , mientras que la validez se hizo a través del juicio de cinco expertos en el área de emprendimiento.

\section{Resultados y discusión}

\subsection{Emprendimientos activos o en etapa de ideación}

En primera instancia se buscó precisar cuántos de los estudiantes encuestados tenían emprendimientos en marcha o al menos en etapa de ideación. En este sentido, se pudo conocer que 47 estudiantes tienen emprendimientos activos (31\%), 58 alumnos están en procesos de ideación de negocios $(38 \%)$, y 47 estudiantes no tienen intención de emprender (31\%). Que la mayoría de los jóvenes manifiesten esta inclinación respalda las ideas de Ortiz (2016) y Barbetti (2020), quienes aseguran que ellos son los principales artífices de la actividad emprendedora. Con base en esto, se hizo un refinamiento de la muestra con el fin de conocer de qué manera la educación recibida en emprendimiento por parte de la institución de educación superior había contribuido positivamente en sus iniciativas; los resultados se muestran a continuación.

\subsection{Influencia de la educación recibida en emprendimiento sobre su acción emprendedora}

A través de la pregunta ¿han influenciado los conocimientos adquiridos en la universidad para desarrollar tus ideas de emprendimiento?, se pudieron conocer los resultados que se aprecian en el Gráfico I.

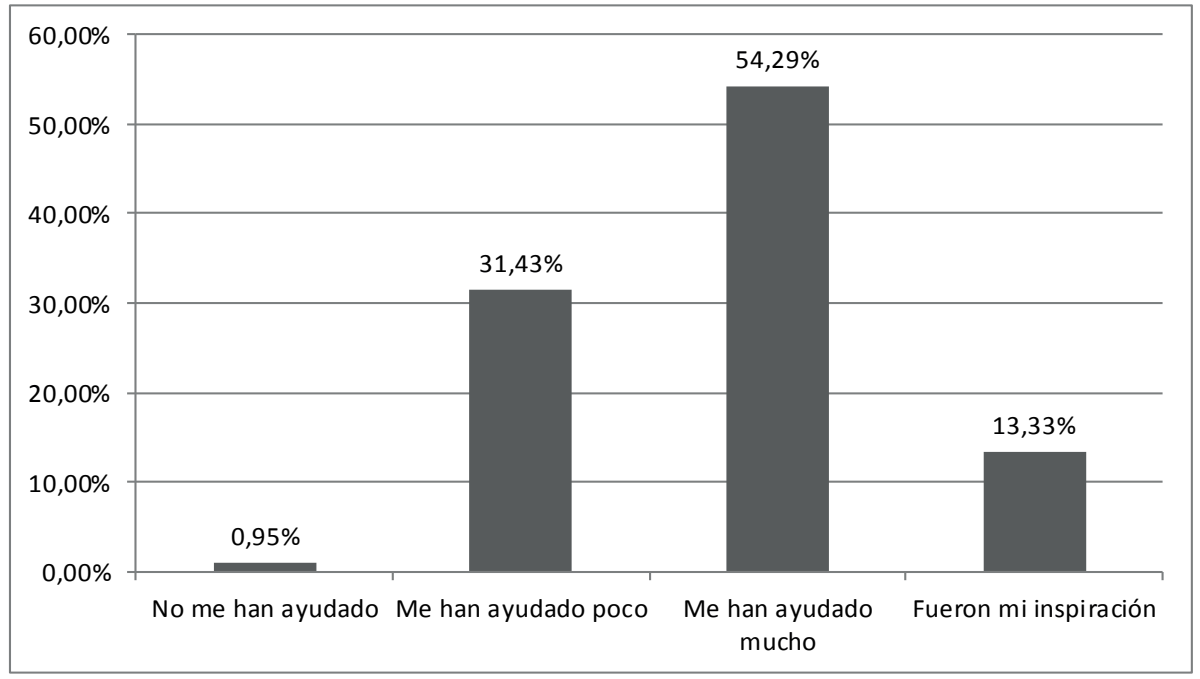

Fuente: Elaboración propia, 2020.

Gráfico I: Influencia de la educación en emprendimiento sobre la acción de emprender 
El Gráfico I, deja en evidencia que la educación recibida en emprendimiento ha influido positivamente en los estudiantes a tal grado que un $13,33 \%$ la considera su inspiración para iniciar sus acciones emprendedoras, un 54,29\% reconoce que le ha sido de mucha ayuda, y un $31,43 \%$ admite que si le ha ayudado aunque no mucho. Sólo un $0,95 \%$ sostiene que esta no le ha ayudado en lo absoluto. Así, queda claro el rol fundamental del docente en la interiorización de la cultura emprendedora, tarea que se ha hecho más ardua a raíz de la virtualización forzada de la educación producto de los confinamientos para evitar la propagación de la COVID-19 (Pineda, Marulanda y Martínez, 2018; MartínezGarcés, Burbano-Vallejo y Burbano-Vallejo, 2019; Martínez-Garcés y Garcés-Fuenmayor, 2020). Asimismo, deja ver cómo la institución de educación superior abordada ha ajustado sus procesos de enseñanza-aprendizaje acorde a las exigencias contemporáneas (Fontalvo y Cienfuegos, 2020).

\subsection{Intenciones emprendedoras}

Para definir las intenciones emprendedoras, se tomó como base la literatura consultada y relacionada en este documento. Tras la recolección de datos a través del cuestionario, el Gráfico II muestra los resultados obtenidos. Es importante aclarar, que para la consolidación de los resultados en este Gráfico, se calcularon las medias aritméticas de cada item, de manera que a las opciones de respuesta les fueron asignados los siguientes intervalos: 1,00 - 1,75 (Ninguna influencia); 1,76 - 2,50 (Poca influencia); 2,51 - 3,15 (Mucha influencia); y 3,16 - 4,00 (Total influencia).

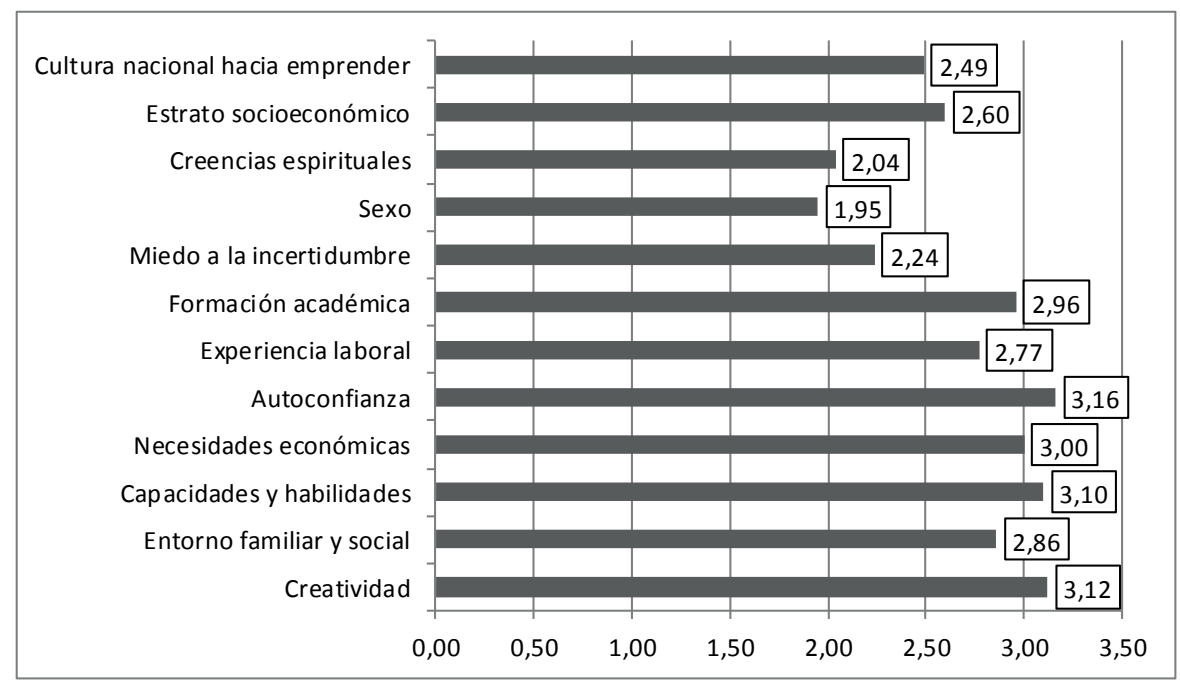

Fuente: Elaboración propia, 2020.

\section{Gráfico II: Intenciones emprendedoras en los estudiantes universitarios}


El Gráfico II, permite determinar las principales intenciones que conllevan a los estudiantes a emprender (aquellas que son $\mu: \geq$ $2,15)$. Estas se pueden agrupar elementalmente en tres categorías: a) Las asociadas a su personalidad, b) las asociadas a su formación y c) las asociadas al medio.

Así, en cuanto a lo personal destaca la creatividad $(\mu: 3,12)$, las capacidades y habilidades $(\mu: 3,10)$, la autoconfianza $(\mu: 3,16)$ y el miedo a la incertidumbre $(\mu: 2,24)$. Estos resultados concuerdan con los planteamientos de Camacho y Viscarra (2020), quienes valoran la creatividad como una de las principales motivaciones para emprender. Esto se relaciona directamente con las capacidades y habilidades (o destrezas de los individuos para transformar las ideas en actos) así como con la innovación y atreverse a correr riesgos (Pulla, González y Macías, 2020).

Por esto, entra en el escenario la autoconfianza para obtener la fortaleza requerida ante cualquier situación complicada por la que puedan atravesar, ingrediente vital para no darse por vencidos ante las primeras pruebas (quizás las más duras en el proceso), y perseverar sin temor al fracaso, el mayor de los miedos de todo emprendedor en sus comienzos (Cajigas, Haro y Ramírez, 2017; Sorroche, 2017). Sin duda, el temor a la incertidumbre que genera la pandemia mundial por la COVID-19, debe ser contrarrestado para darle continuidad a los procesos empresariales.

Sobre su formación, resaltan la académica $(\mu: 2,96)$ y la experiencia laboral $(\mu: 2,77)$. Así, se valida la postura de Hémbuz, Sánchez y Bermeo (2020), quienes mencionan que las universidades colombianas deben fomentar la educación empresarial respondiendo a las necesidades de la sociedad desde la perspectiva de los estudiantes. A esto se le adiciona, la influencia positiva que tienen las experiencias laborales previas, puesto que los estudiantes que han trabajado antes tienen una mayor predisposición a emprender, debido a que tienen un alto control percibido y se sienten mejor formados para crear una empresa (Saavedra y Camarena, 2020).

Finalmente, entre las intenciones asociadas al medio, sobresalen el entorno familiar y social $(\mu: 2,86)$, las necesidades económicas $(\mu: 3,00)$, el estrato socioeconómico $(\mu: 2,60)$ y la cultura nacional hacia emprender $(\mu: 2,49)$. Estos datos son coincidentes con la opinión de Torres-Vázquez, et al. (2020), al señalar que la familia es un elemento clave para la formación en primer plano del espíritu emprendedor junto a los amigos que, en su entorno social, alientan la independencia del joven; por supuesto, esto va de la mano con la carencia de un empleo formal que permita suplir sus necesidades económicas (Socorro, 2020).

Así, se puede notar cómo el estrato socioeconómico sale a relucir en los resultados obtenidos. La población estudiantil de la institución de educación superior estudiada se concentra en los estratos 1,2 y 3 lo que refleja que la intención de emprender se hace más fuerte en los estratos más bajos, tal como afirman Malo (2020); y, Querejazu (2020).

Deahí, que se insista en la cultura nacional de emprendimiento como una estrategia global para la difusión y promoción de esta práctica: En la medida en que exista más confianza en las instituciones y en la economía, más ideas emprendedoras se generarán y llevarán a la realidad. Según Buitrago (2014), la cultura del emprendimiento se apoya en las habilidades, destrezas y saberes que el individuo adquiere socialmente en todos los aspectos relacionados con el proceso emprendedor y con el planteamiento de objetivos de creación de negocios innovadores que contribuyan a fortalecer la sociedad. Así pues, las políticas públicas en el marco de la reactivación económica derivada de la COVID-19, deben apuntar a la garantía del empleo y la vida digna de todos los ciudadanos.

\subsection{Correlaciones de las intenciones no significativas en el proceso emprendedor}

En los resultados expuestos anteriormente se pudo conocer que, de las 
variables estudiadas, solo el sexo $(\mu: 1,95)$ y las creencias espirituales $(\mu: 2,04)$, no resultan significativas al momento de determinar las intenciones emprendedoras, a pesar de que la literatura las señala como importantes del proceso emprendedor. Por ello, se realizó un análisis de correlaciones bivariadas con base al coeficiente Rho de Spearman, con la finalidad de conocer si estas pudieran tener alguna influencia de las otras variables. Los resultados obtenidos se muestran en la Tabla 2.

Tabla 2

Correlaciones

\begin{tabular}{ccccccccccccc}
\hline & IE1 & IE2 & IE3 & IE4 & IE5 & IE6 & IE7 & IE8 & IE9 & IE10 & IE11 & IE12 \\
\hline IE1 & 1,00 & 0,36 & 0,29 & 0,23 &, 120 & 0,32 & 0,28 & 0,37 & 0,31 & 0,39 & 0,34 & 0,4 \\
IE2 & 0,36 & 1,00 & 0,23 & 0,29 &, 043 & 0,37 & 0,22 & 0,33 & 0,33 & 0,34 & 0,52 & 0,32 \\
IE3 & 0,29 & 0,23 & 1,00 & 0,75 & 0,23 & 0,29 &, 160 & 0,35 & 0,32 & 0,28 & 0,31 & 0,28 \\
IE4 & 0,23 & 0,29 & 0,75 & 1,00 &, 189 & 0,31 & 0,25 & 0,35 & 0,37 & 0,28 & 0,36 & 0,29 \\
IE5 &, 120 &, 043 & 0,23 &, 189 & 1,00 &, 071 & 0,27 &, 168 & 0,32 &, 139 & 0,23 &, 080 \\
IE6 & 0,32 & 0,37 & 0,29 & 0,31 &, 071 & 1,00 & 0,47 & 0,66 & 0,45 & 0,7 & 0,49 & 0,71 \\
IE7 & 0,28 & 0,22 &, 160 & 0,25 & 0,27 & 0,47 & 1,00 & 0,65 & 0,42 & 0,55 & 0,4 & 0,49 \\
IE8 & 0,37 & 0,33 & 0,35 & 0,35 &, 168 & 0,66 & 0,65 & 1,00 & 0,48 & 0,84 & 0,61 & 0,68 \\
IE9 & 0,31 & 0,33 & 0,32 & 0,37 & 0,32 & 0,45 & 0,42 & 0,48 & 1,00 & 0,52 & 0,6 & 0,43 \\
IE10 & 0,39 & 0,34 & 0,28 & 0,28 &, 139 & 0,7 & 0,55 & 0,84 & 0,52 & 1,00 & 0,65 & 0,73 \\
IE11 & 0,34 & 0,52 & 0,31 & 0,36 & 0,23 & 0,49 & 0,4 & 0,61 & 0,6 & 0,65 & 1,00 & 0,56 \\
IE12 & 0,4 & 0,32 & 0,28 & 0,29 &, 080 & 0,71 & 0,49 & 0,68 & 0,43 & 0,73 & 0,56 & 1,00 \\
\hline
\end{tabular}

Nota: IE1: Cultura nacional hacia emprender; IE2: Estrato socioeconómico; IE3: Creencias espirituales; IE4: Sexo; IE5: Miedo a la incertidumbre; IE6: Formación académica; IE7: Experiencia laboral; IE8: Autoconfianza; IE9: Necesidades económicas; IE10: Capacidades y habilidades; IE11: Entorno familiar y social; IE12: Creatividad.

Fuente: Elaboración propia, 2020 utilizando Statistical Package for the Social Sciences v.20.

Como se observa en la Tabla 2, las creencias espirituales (IE3) y el sexo (IE4), no presentan correlaciones significativas con el resto de las variables, salvo entre ellas mismas, por lo cual, se convierten en mutuamente descartables dentro del ejercicio: El resultado de 0,75 indica que esta es positivaconsiderable (Mondragón, 2014). El hecho de que el sexo no se correlacione con las variables que resultan más determinantes en el proceso emprendedor se respalda en la caracterización que realiza el Global Entrepreneurship Monitor en Colombia tomando como base el género, donde se conoce que la brecha existente entre la proporción de mujeres emprendedoras es casi la misma que la de hombres emprendedores, si se observa la tasa de actividad empresarial (Gómez, et al., 2019). En este orden de ideas, la visión occidental de la religión tampoco resulta determinante como quizás pueda ocurrir con emprendedores de ascendencia oriental (Torres-Salazar, et al., 2017).

\section{Conclusiones}

Los resultados obtenidos permiten concluir, que entre los estudiantes encuestados existe un alto nivel de espíritu emprendedor el cual se demuestra en iniciativas concretas 
que se encuentran en desarrollo, mientras que otros tienen la intención de iniciarlas. En este sentido, se pudo conocer que la formación en emprendimiento brindada por parte de la institución de educación superior ha contribuido de manera positiva, llegando a ser la inspiración de muchos para cristalizar sus ideas de negocios; esto a pesar de lo difícil que ha sido la virtualización de la educación a partir de la pandemia por COVID-19.

Las intenciones para emprender, se concentran elementalmente en tres aspectos. El primero asociado con lo personal, donde los estudiantes han sido capaces de explorar sus capacidades y habilidades con el fin de explotar su creatividad para tomar la decisión de emprender. Esto ha implicado un fortalecimiento de su autoconfianza con el fin de hacer frente al miedo a la incertidumbre que, de manera natural, genera vivir en la situación de inestabilidad económica mundial debido a la emergencia sanitaria. Por otra parte, algunos aspectos personales que resultaron irrelevantes fueron el sexo y las creencias religiosas.

El segundo, asociado a lo ya dicho, tiene que ver con su formación académica y con el impacto positivo que las experiencias laborales previas tienen sobre su seguridad personal al momento de visualizarse como emprendedores. El tercero y último, es la importancia del entorno en la acción emprendedora: La familia y los amigos, son pieza clave en sus decisiones de iniciar un negocio toda vez que buscan satisfacer sus necesidades económicas, las cuáles son significativas por pertenecer mayormente a estratos socioeconómicos 1, 2 y 3. En este sentido, el apoyo gubernamental al fomento de una cultura emprendedora, es de vital importancia para generar confianza en la economía y las instituciones.

\section{Referencias bibliográficas}

Arias, C. M., y Castillo, E. (2011). La educación para el emprendimiento y empresarismo virtual: Potencialidades. Revista Virtual Universidad Católica

$$
\text { del Norte, (32), 1-8. }
$$

Avendaño, W. R., Luna, H. O., y Quintero, L. (2020). La política colombiana de emprendimiento en educación y su impacto en el acceso al empleo de jóvenes. El Ágora USB, 20(2), 158-171. https://doi. org/10.21500/16578031.5137

Barbetti, P. A. (2020). Promoción de emprendimientos y autoempleo para jóvenes en políticas y programas de Argentina. Revista de Ciencias Sociales (Ve), XXVI(1), 272286. https://doi.org/10.31876/rcs. v26i1.31324

BC Noticias (4 de enero de 2021). Colombia arranca el 2021 con la nueva Ley de Emprendimiento. BC Noticias. https:// www.bcnoticias.com.co/colombiaarranca-el-2021-con-la-nueva-ley-deemprendimiento/

Borrayo, C. L., Valdez, A., y Delgado, B. (2019). Cultura emprendedora en jóvenes universitarios de Guadalajara, México. Revista de Ciencias Sociales (Ve), $X X V(3), 72-87$.

Buitrago, J. A. (2014). Emprendimiento en Colombia. Administración \& Desarrollo, 43(59), 7-21.

Cajigas, M., Haro, M. D. C., y Ramírez, E. (2017). El estado colombiano y el emprendimiento empresarial: éxito o fracaso de su programa clave. Criterio Libre, 15(26), 105-130. https://doi.org/10.18041/1900-0642/ criteriolibre. $2017 \mathrm{v} 15 \mathrm{n} 26.1043$

Camacho, W. R., y Viscarra, C. P. (2020). Factores que influyen en la creatividad de una persona para identificar oportunidades de negocio en el cantón de Guaranda, provincia de Bolívar en el año 2020. Enlace Universitario, 19(2), 85-93. https://doi.org/10.33789/ enlace.19.2.76

Cardona, D., Montenegro, A., y Hernández, 
H. G. (2017). Creación de empresa como pilar para el desarrollo social e integral de la región Caribe en Colombia: apuntes críticos. Saber Ciencia y Libertad, 12(1), 134-143. https://doi.org/10.18041/2382-3240/ saber.2017v12n1.698

Chicas, S. M. (2020). La educación superior virtual y su influencia en el emprendimiento en Colombia: Revisión de literatura. Working Papers: Negocios, Gestión y Sostenibilidad, 1(1), 1-12. https://doi. org/10.15765/wp.v1i1.1572

Contreras, A., y Macías, P. (2021). Percepción de los estudiantes sobre la intención de emprender. Caso: Universidad de Guadalajara, México. SUMMA. Revista Disciplinaria en Ciencias Económicas y Sociales, 3(1), 1-25. $\quad$ https://doi.org/10.47666/ summa.3.1.03

Díaz, A. M., y Silva, A. C. (2017). Retos de las políticas públicas para el fomento del emprendimiento femenino en Colombia. Reflexión Política, 19(38), 42-57. https://doi. org/10.29375/01240781.2838

Durán, S., y Martínez, J. (2019). Intenciones emprendedoras en estudiantes universitarios. Avances en Investigación Científica, 1(1), 22-23.

Fontalvo, W., y Cienfuegos, R. (2020). Características del liderazgo innovador que impulsan la cultura de la innovación en las unidades de emprendimiento de las instituciones de educación superior del departamento del Atlántico. Dictamen Libre, (27), 35-49. https://doi.org/10.18041/26194244/dl.27.6643

Gómez, L., López, S., Hernández, N., Galvis, M., Parra, F. J., Matiz, R., Varela, J. A., Moreno, F., Pereira, A., Arias, G., García, C., y Martínez, P. C. (2019). GEM Colombia. Estudio de la actividad empresarial en 2017. Editorial Universidad del Norte.

Hémbuz, G. D., Sánchez A. M., y Bermeo, V. (2020). Influencia de la educación superior en el emprendimiento juvenil en estudiantes universitarios: Una aproximación teórica. Boletín Redipe, 9(8), 166-180. https://doi. org/10.36260/rbr.v9i8.1049

Hinojosa, E. (18 de enero de 2021). La pandemia $\mathrm{y}$ el auge del emprendimiento. Razonpublica. https://razonpublica. com/la-pandemia-auge-delemprendimiento/

Malo, M. (2020). Breve recorrido por el emprendimiento social en Japón: Del Japón pre-moderno hasta el Tōhoku post Fukushima. Mirai. Estudios Japoneses, 4, 75-85. https://doi. org/10.5209/mira.67537

Martínez-Garcés, J.., Burbano-Vallejo, M. y Burbano-Vallejo, E. (2019). Obstáculos y perspectivas al emplear tecnologías de información para enseñar contabilidad. Educación y Humanismo, 21(37), 104-119. https:// doi.org/10.17081/eduhum.21.37.3461

Martínez-Garcés, J., y Garcés-Fuenmayor, J. (2020). Competencias digitales docentes y el reto de la educación virtual derivado de la covid-19. Educación y Humanismo, 22(39), 1-16. $\quad$ https://doi.org/10.17081/ eduhum.22.39.4114

Ministerio de Salud y Protección Social de la República de Colombia (6 de marzo de 2020). Colombia confirma su primer caso de COVID-19. MinSalud. https://www.minsalud.gov.co/Paginas/ Colombia-confirma-su-primer-casode-COVID-19.aspx

Mondragón, M. A. (2014). Uso de la correlación de Spearman en un estudio de intervención en fisioterapia. Movimiento Científico, 8(1), 98-104. 
https://doi.org/10.33881/2011-7191. mct.08111

Muñóz, Á., y Martínez, L. (2020). Emprendimiento social y felicidad urbana. SUMMA. Revista Disciplinaria en Ciencias Económicas y Sociales, 2(1), 127-169.

Ortiz, P. (2016). Actitud emprendedora de los jóvenes españoles en un contexto de crisis. Revista de Ciencias Sociales (Ve), XXII(1), 139-152.

Patiño, J. D., Ruiz, A., y Pitre-Redondo, R. (2018). El emprendimiento en Colombia, una respuesta a los retos de competitividad y desarrollo sostenible. Revista Espacios, 39(14), 24-34.

Pérez, C., y Álvarez, Á. (2017). La inclusión laboral y la generación de emprendimientos sociales en comunidades vulnerables: el caso de la Urbanización Altos de la Sabana en la ciudad de Sincelejo - Colombia. En J. J. Hernández (Ed.), Transdisciplina y complejidad. Aportes de las ciencias sociales en América latina, el Caribe y Colombia al desarrollo social (pp. 109-139). Editorial CECAR. https:// doi.org/10.21892/9789588557380

Pineda, L., Marulanda, C., y Martínez, J. (2018). Fomento del emprendimiento cultural en instituciones de educación superior ubicadas en Santiago de Cali - Colombia. Encuentro Educacional, 25(1), 38-55.

Pulla, E. V., González, E. X., y Macías, B. C. (2020). Competencias personales. Análisis de las habilidades y capacidades de los emprendedores. Revista Espacios, 41(32), 248-263.

Querejazu, V. (2020). Hacia un modelo de la conducta emprendedora en Bolivia. Nóesis, 29(57), 1-22. http://dx.doi. org/10.20983/noesis.2020.1.1

Rico, A. Y., y Santamaría, M. (2017). Análisis comparativo de los procesos existentes en el campo del emprendimiento en la educación media en Colombia y Ecuador. Voces y Silencios: Revista Latinoamericana de Educación, 8(2), 53-68.

Rodríguez, C. A., y Prieto, F. A. (2009). La sensibilidad al emprendimiento en los estudiantes universitarios. Estudio comparativo ColombiaFrancia. Innovar. Revista de Ciencias Administrativas y Sociales, $19(\mathrm{~S}-1)$, 73-89.

Saavedra, M. L., y Camarena, M. E. (2020). Intención de emprendimiento en los estudiantes de la Ciudad de México. RAN - Revista Academia \& Negocios, 5(2), 85-98.

Semana (13 de noviembre de 2020). Quiebra empresarial. Cómo ha afectado la pandemia la operación de las empresas en Colombia. Semana. https://www. semana.com/empresas/articulo/ cuantas-empresas-han-quebrado-encolombia-por-la-pandemia/306739/

Socorro, F. (2020). Emprendimientos y microempresas: las sutiles diferencias de dos grandes herramientas. Ámbito Investigativo, 5(1), 15-22.

Solis, J. B., Neira, M. L., Ormaza, J. E., y Quevedo, J. (2021). Emprendimiento e innovación: Dimensiones para el estudio de las MiPymes de AzoguesEcuador. Revista de Ciencias Sociales (Ve), XXVII(1), 315-333.

Sorroche, R. (2017). Aprende a emprender (Tesis de pregrado). Universidad de Sevilla, Sevilla, España.

Suárez, M., Suárez, L., y Zambrano, S. M. (2017). Emprendimiento de jóvenes rurales en Boyacá - Colombia: Un compromiso de la educación y los gobiernos locales. Revista de Ciencias Sociales (Ve), XXIII(4), 23-32.

Tarapuez, E., Osorio, H., y Botero, J. J. (2013). Política de emprendimiento 
en Colombia 2002-2010. Estudios Gerenciales, 29(128), 274-283. https:// doi.org/10.1016/j.estger.2013.09.001

Torres-Salazar, P., Flórez-Donado, J., MorganBeltrán, J., Rodríguez-Calderón, G., Melamed-Varela, E., Espinosa, V. M., y Niño, J. L. (2017). Estudio de influencia de la cultura en el emprendimiento: caso de la comunidad árabe de Barranquilla, Colombia.
Revista Espacios, 38(41), 20-38.

Torres-Vázquez, K. F., Erazo-Álvarez, J. C., Narváez-Zurita, C. E., y PaulinaMoreno, V. (2020). El emprendimiento juvenil en función al perfil psicosocial y las condiciones del entorno familiar. Revista Arbitrada Interdisciplinaria Koinonia, 5(10), 729-756. http:// dx.doi.org/10.35381/r.k.v5i10.714 